\title{
OPEN Repetitive visual cortex transcranial random noise stimulation in adults with amblyopia
}

\author{
Richard Donkor ${ }^{1}$, Andrew E. Silva ${ }^{1}$, Caroline Teske ${ }^{1}$, Margaret Wallis-Duffy ${ }^{1}$, \\ Aaron P. Johnson ${ }^{2,3,4}$ \& Benjamin Thompson ${ }^{1,5,6 \rrbracket}$
}

We tested the hypothesis that five daily sessions of visual cortex transcranial random noise stimulation would improve contrast sensitivity, crowded and uncrowded visual acuity in adults with amblyopia. Nineteen adults with amblyopia ( $44.2 \pm 14.9$ years, 10 female) were randomly allocated to active or sham tRNS of the visual cortex (active, $n=9$; sham, $n=10$ ). Sixteen participants completed the study ( $n=8$ per group). tRNS was delivered for 25 min across five consecutive days. Monocular contrast sensitivity, uncrowded and crowded visual acuity were measured before, during, 5 min and 30 min post stimulation on each day. Active tRNS significantly improved contrast sensitivity and uncrowded visual acuity for both amblyopic and fellow eyes whereas sham stimulation had no effect. An analysis of the day by day effects revealed large within session improvements on day 1 for the active group that waned across subsequent days. No long-lasting (multi-day) improvements were observed for contrast sensitivity, however a long-lasting improvement in amblyopic eye uncrowded visual acuity was observed for the active group. This improvement remained at 28 day follow up. However, between-group differences in baseline uncrowded visual acuity complicate the interpretation of this effect. No effect of tRNS was observed for amblyopic eye crowded visual acuity. In agreement with previous non-invasive brain stimulation studies using different techniques, tRNS induced short-term contrast sensitivity improvements in adult amblyopic eyes, however, repeated sessions of tRNS did not lead to enhanced or long-lasting effects for the majority of outcome measures.

Amblyopia is a developmental disorder of the visual cortex, with a prevalence of approximately $1-5 \%^{1-3}$. Amblyopia causes a wide range of vision deficits ${ }^{4,5}$ including a monocular loss of high-contrast visual acuity $y^{6,7}$ that is particularly pronounced for crowded optotypes ${ }^{8,9}$, reduced contrast sensitivity in the affected eye $e^{6,10-12}$, and impaired or absent stereopsis ${ }^{13,14}$. Amblyopia is also associated with chronic suppression of the affected eye $e^{15,16}$ that may play a key role in the etiology of the disorder ${ }^{17}$.

Amblyopia involves abnormal processing within the primary and extrastriate visual cortex ${ }^{18}$ and therefore recovery from amblyopia requires a change in cortical function. Current amblyopia treatments achieve this by directly manipulating visual input to the brain. For example, the most common amblyopia treatment involves the provision of a clear retinal image in the amblyopic eye using refractive correction followed by occlusion of the non-amblyopic eye. This treatment improves amblyopic eye visual acuity, but has drawbacks in terms of compliance $^{19}$ and reduced efficacy with increasing age $\mathrm{e}^{20}$.

Transcranial electrical stimulation (tES) refers to a suite of non-invasive neuro-modulation techniques including transcranial direct current stimulation (tDCS), transcranial alternating current stimulation (tACS) and transcranial random noise stimulation (tRNS) that may enhance plasticity in targeted regions of the human brain ${ }^{21-23}$, including the visual cortex ${ }^{24-26}$. Currently, tES methods are being investigated as a potential neurorehabilitation tool for disorders including stroke ${ }^{27-30}$, chronic pain ${ }^{31,32}$ and tinnitus ${ }^{33,34}$ and there is growing interest in the use of tES and transorbital stimulation to treat disorders of vision ( $\mathrm{see}^{35-38}$ for recent reviews).

Following early work that reported improved contrast sensitivity in adults with amblyopia after visual cortex repetitive transcranial magnetic stimulation ${ }^{39,40}$, a number of studies have investigated the application of

\footnotetext{
${ }^{1}$ Department of Optometry and Vision Science, University of Waterloo, 200 University Avenue West, Waterloo, ON NOB 2TO, Canada. ${ }^{2}$ Department of Psychology, Concordia University, Montreal, Canada. ${ }^{3}$ Réseau de Recherche en Santé de la Vision, Montreal, Canada. ${ }^{4}$ CRIR/Lethbridge-Layton-Mackay Rehabilitation Centre du CIUSSS du Ce ntre-Ouest-de-l'Île-de-Montréal, Montreal, Canada. ${ }^{5}$ Center for Eye and Vision Research, Hong Kong, China. ${ }^{6}$ The Liggins Institute, University of Auckland, Auckland, New Zealand. ${ }^{\boxplus}$ email: ben.thompson@uwaterloo.ca
} 
anodal tDCS to amblyopia. A single session of anodal tDCS improves amblyopic eye contrast sensitivity ${ }^{41,42}$, increases visually evoked potential (VEP) amplitudes induced by stimuli presented to the amblyopic eye ${ }^{42}$, and balances the response to inputs from each eye within visual cortex ${ }^{41}$. Furthermore, anodal tDCS enhances the effect of perceptual learning (PL) in adults with amblyopia ${ }^{26}$ and recent studies have revealed that visual acuity, detection thresholds, and stereopsis improve in mature amblyopic rats following anodal $\mathrm{tDCS}{ }^{43-45}$. One potential mechanism for anodal tDCS effects in adults with amblyopia is a reduction in GABA-mediated inhibition ${ }^{46}$ within the visual cortex. GABA has been associated with interocular suppression in strabismic cats ${ }^{47}$ and may act as a "break" on visual cortex plasticity ${ }^{48}$.

A recently developed tES technique, $\mathrm{tRNS}$, involves an alternating current that randomly changes in frequency and amplitude ${ }^{49}$. tRNS may have larger effects on cortical activity than other tES protocols. For example, tRNS induced significantly greater improvements in tinnitus symptoms ${ }^{33}$ and larger increases in motor evoked potential amplitude (MEP) $)^{21}$ compared to either tDCS or tACS. Furthermore, visual cortex tRNS enhanced visual perceptual learning in adults with normal vision to a greater extent than anodal $t D C S^{50,51}$ and the combination of tRNS and perceptual learning enhanced the transfer of learning to non-trained visual tasks in adults with amblyopia ${ }^{24}$. tRNS has also been reported to enhance visual perceptual learning in adults with cortical blindness ${ }^{51}$. Potential mechanisms for these effects include an acute enhancement of the signal-to-noise ratio within the visual cortex due to stochastic resonance $e^{52-54}$ and longer-lasting alterations in neural membrane function due to repetitive opening and closing of sodium channels ${ }^{55}$.

Within this single-blind, between subjects, randomized, sham-controlled study, we tested the hypothesis that five daily sessions of visual cortex tRNS alone would lead to improved amblyopic eye contrast sensitivity, along with improved crowded and uncrowded amblyopic eye visual acuity in adult participants.

\section{Methods and materials}

Participants. Amblyopia was defined as reduced best corrected visual acuity $(>+0.3 \operatorname{logMAR})$ in one eye in the absence of ocular pathology and at least a $0.2 \log M A R$ acuity difference between the eyes. Anisometropia was defined as a difference in spherical equivalent between the two eyes of $\geq 0.50$ Diopters (D), or a difference of astigmatism in any meridian $\geq 1.50 \mathrm{D}^{56}$. Initial visual acuity was measured using an M\&S logMAR chart. Inclusion criteria were: (i) presence of strabismic and/or anisometropic amblyopia; (ii) 0.0 logMAR visual acuity or better in the fellow eye (FE). Exclusion criteria ${ }^{57-60}$ were: (i) presence of a scalp skin condition that contraindicated tRNS; (ii) history of neurological or psychiatric disorders, such as seizures; (iii) current medication for the treatment of neurological or psychiatric disorders; (iv) a history of brain injury; (v) implanted medical devices. Potential participants were contacted following a search of the patient database at Dr. Teske and Associates. Interested participants completed telephone screening to determine eligibility. The experimental procedures were approved by the Ethics Review Board of the University of Waterloo, Canada and were consistent with the declaration of Helsinki. Written informed consent was obtained from all participants. All participants were remunerated for their time.

Procedure. A single-blind, sham controlled, between-subjects design was adopted. Randomization followed allocation concealment procedures and was conducted by an experimenter who was not involved in data collection or eligibility assessment using a random number generator. Randomization occurred after participants had met the eligibility criteria and completed study enrolment. Participants completed 5 consecutive daily stimulation sessions and a follow-up session 28 days after the final stimulation session. Outcome measures were completed by the participants using automated computer programs with no input from the experimenter. This procedure was designed to mitigate against experimenter bias.

Transcranial random noise stimulation. Subjects either received tRNS $(2.0 \mathrm{~mA}$, current density: $0.08 \mathrm{~mA} / \mathrm{cm}$, frequency range $0.1-640 \mathrm{~Hz}$ ) or placebo (sham) stimulation of the primary visual cortex for 25 min over 5 consecutive days. Stimulation was delivered using a DC-stimulation MC device (Eldith, NeuroConn $\mathrm{GmbH}$, Germany). We chose a relatively long and high intensity stimulation protocol because we planned to measure 6 outcomes (contrast sensitivity, crowded and uncrowded visual acuity for each eye) and therefore attempted to maximize the duration of the stimulation aftereffects ${ }^{61,62}$. There is no statistically significant difference between the effect of high frequency $(101-640 \mathrm{~Hz})$ and low frequency $(0.1-100 \mathrm{~Hz})$ visual cortex tRNS on visual perceptual learning enhancement ${ }^{50}$. Therefore, we chose to deliver the full frequency range. The stimulation was delivered via a pair of saline-soaked surface sponge electrodes $\left(5 \mathrm{~cm} \times 5 \mathrm{~cm}, 25 \mathrm{~cm}^{2}\right)$ placed at $\mathrm{Cz}$ and $\mathrm{Oz}^{63}$, as determined by the international 10/20 electroencephalogram system. The AC current was initially ramped up to a maximum of $2 \mathrm{~mA}$ over $30 \mathrm{~s}$ and ramped down to $0 \mathrm{~mA}$ over $30 \mathrm{~s}$ at the end of the stimulation session. During sham stimulation, the $30 \mathrm{~s}$ ramp-up was immediately followed by the ramp-down out ${ }^{64}$. Our between subjects design and use of participants entirely naïve to non-invasive brain stimulation ensured that participants remained masked to their treatment allocation. Our application of tRNS conformed to tDCS safety guidelines ${ }^{57,65}$.

After the final tRNS session, participants were asked to rate the following sensations on a four-level scale (none, mild, moderate and severe): headache, neck pain, scalp pain, tingling, itching, burning sensation, sleepiness, trouble concentrating and acute mood change. Participants were also asked to rate whether any reported sensations were due to tES by selecting from the following options: no, remote chance, probable, definitely.

Visual function measurements. Monocular contrast sensitivity and visual acuity (both crowded and uncrowded) were measured for each eye before, during, $5 \mathrm{~min}$ post, and $30 \mathrm{~min}$ post stimulation on each stimulation day (Fig. 1). All measurements were made using Landolt-C optotypes presented using the Freiburg Vision 

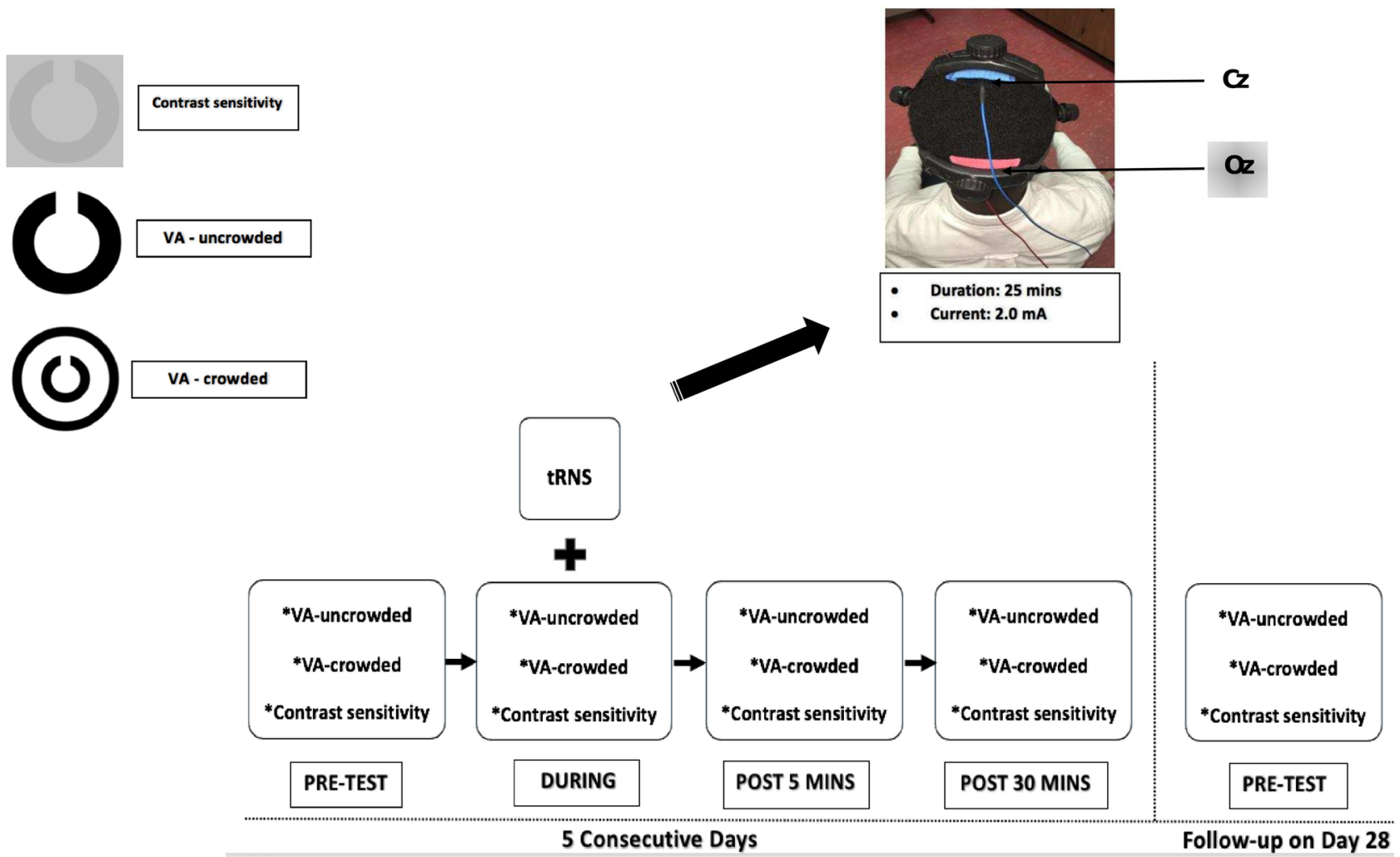

Figure 1. Testing and stimulation protocol. Each measurement was recorded before stimulation (pre-test), during stimulation, $5 \mathrm{~min}$ after stimulation (Post $5 \mathrm{~min}$ ) and $30 \mathrm{~min}$ after stimulation (Post $30 \mathrm{~min}$ ) for 5 consecutive days. Baseline (pre-test) measurements were recorded again for each eye 28 days (Day 28) after the last stimulation session. Stimulation was delivered for $25 \mathrm{~min}$ at $2.0 \mathrm{~mA}$. Active and reference electrodes were placed at $\mathrm{Oz}$ and $\mathrm{Cz}$ respectively. $V A$ visual acuity.

Test ('FrACT') ${ }^{66,67}$ software package on a MacBook Pro (Version 10.13.6, 13-in, 2.7 GHz, 2560× 1600). Gamma correction was conducted using a Spyder photometer and the FrACT software provided 10 bits of contrast resolution. The Landolt- $\mathrm{C}$ optotype was presented at 8 possible orientations and viewed from $3 \mathrm{~m}$ in a dark room. Participants identified the gap orientation using button presses. Trials were self-paced with a maximum display time of $30 \mathrm{~s}$. A Bayesian adaptive ("Best PEST") algorithm controlled optotype size for the crowded and uncrowded visual acuity threshold measurements and optotype contrast for the contrast sensitivity threshold measurement. Each threshold measurement lasted approximately $3 \mathrm{~min}$. For the measurements made during stimulation, threshold measurement started $5 \mathrm{~min}$ into the stimulation. Landolt-C gap width was fixed at 30 arcmin for the non-amblyopic eye and 100 arcmin for the amblyopic eye during the contrast sensitivity measures. These parameters were based on pilot observations in individuals with moderate and severe amblyopia who could not resolve the 30 arcmin stimuli. Crowded optotypes were surrounded by a circle. Both the fellow eye and amblyopic eye were tested monocularly with the fellow eye tested first within each block. Uncrowded visual acuity was tested first within each block followed by crowded visual acuity and contrast sensitivity.

Data analysis. Statistical analyses were performed in R (R Core Team, 2020) ${ }^{68}$ using the Bootstrap-Coupled Estimation package ${ }^{69}$. Visual acuities were recorded in logMAR units. Contrast sensitivity was recorded in log units. To test for tRNS effects within the 5 stimulation sessions, a mixed-effects analysis of variance (ANOVA) with a between-subjects factor of Group (active vs sham), a within-subjects factor of Day (day 1-5), and a within-subjects factor of Time (baseline, during, post 5 and post $30 \mathrm{~min}$ ) was conducted for each measurement type for each eye separately. Planned pairwise comparisons (least significance difference test) between baseline and all other timepoints were examined for each day. In addition, to assess whether tRNS had cumulative or long-term effects on visual function, a mixed ANOVA with factors of Group (active vs sham) and Baseline (baseline day 1, baseline day 2, baseline day 3, baseline day 4, baseline day 5, baseline day 28) was conducted for each outcome measure for each eye. All ANOVA analyses reported passed Levene's test for equality of variances $(\mathrm{p}>0.05)$ and test of sphericity $(\mathrm{p}>0.05)$.

Pairwise comparisons were conducted using the effect size Hedge's $g$ by bootstrap estimation (5000 bootstrap samples with replacement), with the $95 \%$ confidence interval around the $g$ being bias-corrected and accelerated ${ }^{69}$. The permutation $p$ values reported were calculated with 5000 reshuffles of the baseline and test labels performed for each permutation, with the $\mathrm{P}$ value indicating the likelihood of observing the mean difference, if the null hypothesis of zero difference is true, at an $\alpha$ of 0.05 . Between group differences in the strength of any sensations induced by tRNS were assessed using the chi-squared test. 


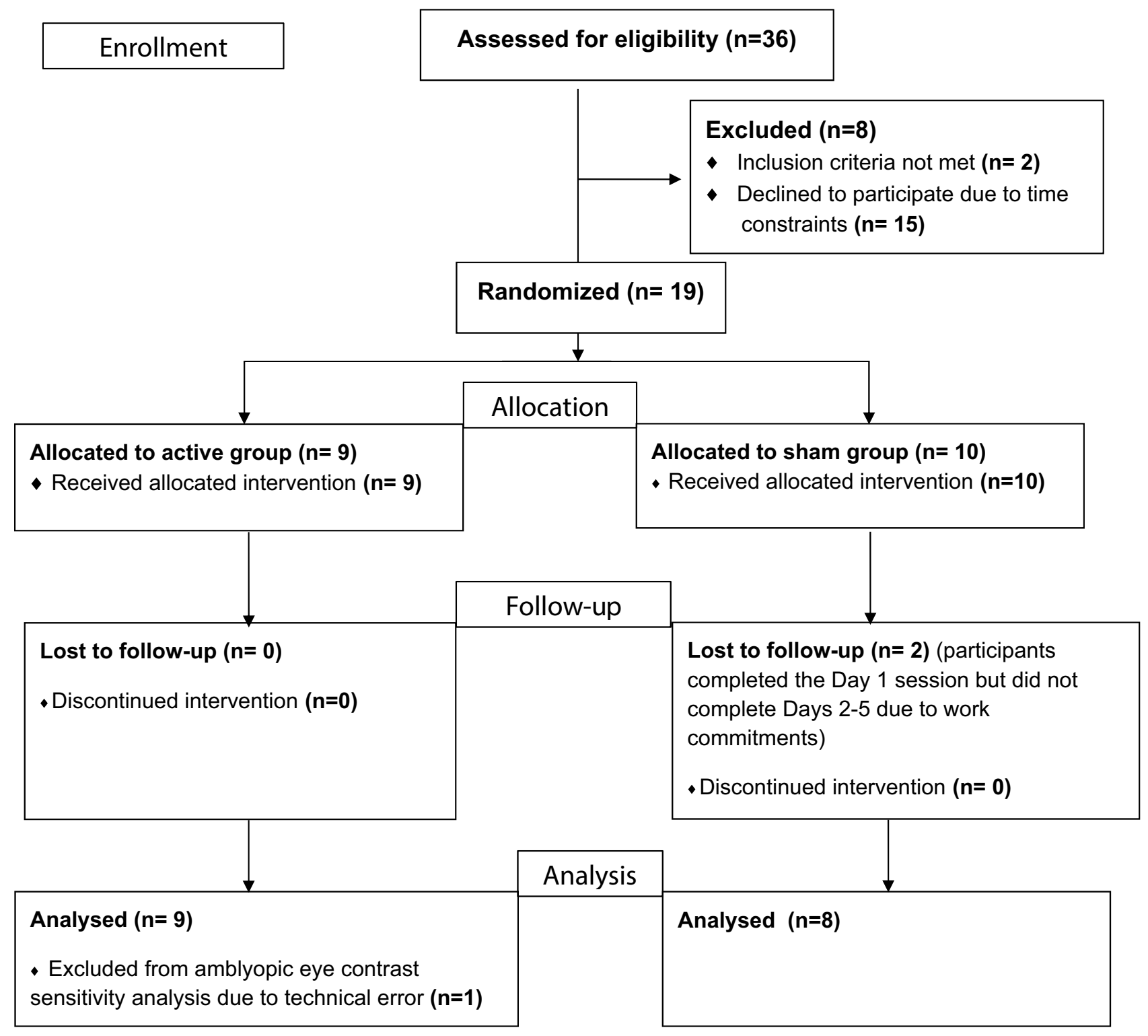

Figure 2. CONSORT flow diagram for the study.

\section{Results}

Nineteen healthy adults with amblyopia were recruited. Two participants in the sham group withdrew from the study after day 1 due to the time commitment required and were excluded from the analysis (Fig. 2). Clinical details of the remaining seventeen participants are provided in (Table 1). A technical error prevented an accurate amblyopic eye contrast sensitivity measurement on day 1 for one participant in the active group. This participant was excluded from the amblyopic eye contrast sensitivity analysis only (Fig. 2). There was no statistically significant group difference in age for the 17 participants that completed the study (Active group mean age $=38.7$ years, $\mathrm{SD}=13.6$; Sham group mean age $=45.8, \mathrm{SD}=15.4 ; \mathrm{t}_{15}=1.0, \mathrm{p}=0.3$ ). There were no adverse effects of $\mathrm{tRNS}$, and there were no statistically significant between-group differences in the range or severity of subjective sensations reported (Table 2).

Contrast sensitivity. For the amblyopic eyes (Fig. 3-upper panel), there was a significant interaction between Group and Time, $\mathrm{F}_{3,42}=3.584, \mathrm{p}=0.022, \eta_{\mathrm{p}}{ }^{2}=0.216$. No other omnibus main effects or interactions were significant. Planned pairwise comparisons between baseline and all other timepoints were examined for the active and sham groups for each day. During day 1, the active group exhibited a significant improvement in contrast sensitivity from baseline for all post-test measurements (during: $g=0.272$ [0.195, 0.597], $\mathrm{p}=0.01$; post 5 min: $g=0.236[0.039,0.726], \mathrm{p}=0.035$; and post $30 \mathrm{~min}: g=0.438[0.052,1.207], \mathrm{p}=0.034$ : Fig. 4$)$. No significant differences between baseline and any post-test were found for days $2-5$. No significant differences between baseline and any post-test were found within the sham group for any day.

For the fellow eyes (Fig. 3-lower panel), there was a significant interaction between Group and Time, $\mathrm{F}_{3,45}=3.303, \mathrm{p}=0.029, \eta_{\mathrm{p}}{ }^{2}=0.191$. No other omnibus main effects or interactions were significant. During day 


\begin{tabular}{|c|c|c|c|c|c|c|c|c|c|}
\hline \multirow[b]{2}{*}{ Participant } & \multirow[b]{2}{*}{ Age/sex } & \multirow[b]{2}{*}{ Patching } & \multirow{2}{*}{$\begin{array}{l}\text { Type of } \\
\text { amblyopia }\end{array}$} & \multirow[b]{2}{*}{ Stereoacuity } & \multicolumn{2}{|c|}{$\begin{array}{l}\text { Visual acuity } \\
\text { (logMAR) }\end{array}$} & \multicolumn{3}{|l|}{ Current refraction } \\
\hline & & & & & AME & FFE & AME & FFE & ADD \\
\hline Active 1 & $27 / \mathrm{M}$ & Yes & Mixed & $<800$ & +1.40 & 0.00 & $+2.00-0.50 \times 155$ & $+0.50 \mathrm{DS}$ & \\
\hline Active 2 & $20 / \mathrm{M}$ & Yes & Aniso & 400 & +0.50 & 0.00 & $+1.00-1.50 \times 175$ & Plano & \\
\hline Active 3 & $37 / \mathrm{M}$ & Yes & Aniso & $<800$ & +0.30 & 0.00 & $-1.00-1.00 \times 100$ & $\begin{array}{l}-0.25- \\
2.50 \times 090\end{array}$ & \\
\hline Active 4 & $45 / \mathrm{F}$ & Yes & Aniso & 200 & +0.30 & 0.00 & $+2.75-0.75 \times 080$ & $\begin{array}{l}+2.00- \\
0.25 \times 159\end{array}$ & +1.50 \\
\hline Active 5 & $59 / \mathrm{M}$ & No & Aniso & $<800$ & +0.70 & 0.00 & $+8.25 \mathrm{DS}$ & $\begin{array}{l}+8.25- \\
1.25 \times 105\end{array}$ & +2.50 \\
\hline Active 6 & $41 / \mathrm{F}$ & Yes & Mixed & $<800$ & +0.50 & 0.00 & $+2.00 \mathrm{DS}$ & $\begin{array}{l}+0.25- \\
3.75 \times 179\end{array}$ & +0.75 \\
\hline Active 7 & $46 / \mathrm{M}$ & No & Aniso & $<800$ & +0.40 & 0.00 & $+0.75 \mathrm{DS}$ & $\begin{array}{l}+4.25 \\
-0.50 \times 165\end{array}$ & \\
\hline Active 8 & $52 / \mathrm{F}$ & No & Aniso & 400 & +0.70 & 0.00 & $+0.50-0.50 \times 175$ & $\begin{array}{l}+2.00- \\
0.50 \times 075\end{array}$ & +2.25 \\
\hline Active $9^{*}$ & $21 / \mathrm{F}$ & No & Aniso & 60 & +0.50 & +0.10 & $+0.50-4.00 \times 180$ & Plano & \\
\hline Sham 1 & $51 / \mathrm{F}$ & Yes & Mixed & $<800$ & +0.30 & 0.00 & $+3.25-0.50 \times 165$ & Plano & \\
\hline Sham 2 & $58 / \mathrm{M}$ & Yes & Aniso & 400 & +0.30 & 0.00 & $+4.00-0.25 \times 180$ & $\begin{array}{l}+1.50- \\
0.50 \times 180\end{array}$ & +2.25 \\
\hline Sham 3 & $52 / \mathrm{F}$ & No & Aniso & 200 & +0.40 & 0.00 & $-2.50-0.50 \times 155$ & $-1.50 \mathrm{DS}$ & +2.75 \\
\hline Sham 4 & $19 / \mathrm{M}$ & No & Aniso & $<800$ & +0.30 & 0.00 & $+2.25 \mathrm{DS}$ & $+0.75 \mathrm{DS}$ & \\
\hline Sham 5 & $55 / \mathrm{M}$ & No & Aniso & $<800$ & +0.30 & 0.00 & $+1.75-0.75 \times 085$ & $\begin{array}{l}+1.75- \\
0.75 \times 090\end{array}$ & +2.00 \\
\hline Sham 6 & $49 / \mathrm{M}$ & No & Strab & 100 & +0.30 & 0.00 & $+1.75 \mathrm{DS}$ & $+1.50 \mathrm{DS}$ & \\
\hline Sham 7 & $24 / \mathrm{M}$ & No & Mixed & $<800$ & +0.30 & 0.00 & $+8.25-2.00 \times 160$ & $\begin{array}{l}+6.50- \\
1.25 \times 0.40\end{array}$ & \\
\hline Sham 8 & $58 / \mathrm{F}$ & No & Strab & $<800$ & +0.50 & 0.00 & $+4.50-0.25 \times 176$ & $\begin{array}{l}+3.50- \\
0.50 \times 165\end{array}$ & +2.75 \\
\hline
\end{tabular}

Table 1. Participant details. $\mathrm{M}=$ male, $\mathrm{F}=$ female, patching = previous history of occlusion therapy, mixed $=$ mixed amblyopia, aniso = anisometropic amblyopia, strab = strabismic amblyopia, stereoacuity is presented in seconds of arc, $\mathrm{AME}=$ amblyopic eye, $\mathrm{FFE}=$ fellow fixating eye, add = near power addition. ${ }^{\star}$ Indicates the participant with missing data for the amblyopic eye contrast sensitivity measurement only.

1, the active group exhibited a significant improvement in contrast sensitivity from baseline for the post 5 min $(g=0.639[0.127,1.248], \mathrm{p}=0.033)$ and post $30 \mathrm{~min}(g=0.846[0.199,1.661], \mathrm{p}=0.018)$ measurements. No significant differences between baseline and any post-test were found for days $2-5$. No significant differences between baseline and any post-test were found within the sham group for any day.

Uncrowded visual acuity. For the amblyopic eyes (Fig. 5-upper panel), there was a significant interaction between Group and Time, $F_{3,45}=3.325 ; \mathrm{p}=0.029, \eta_{\mathrm{p}}{ }^{2}=0.192$ ). No other omnibus main effects or interactions were significant. During day 1 , the active group exhibited a significant improvement in uncrowded visual acuity from baseline for all post-test measurements (during: $g=0.224$ [0.084, 0.575], $\mathrm{p}=0.010$; post 5: $g=0.281$ $[0.009,0.640], \mathrm{p}=0.05$; post $30: \mathrm{g}=0.307$ [0.118, 0.795], $\mathrm{p}=0.003)$. During days 2 and 3 , the active group exhibited a significant difference between baseline and only the post $5 \mathrm{~min}$ measurement (day 2: $g=0.231$ [0.091, $0.383], \mathrm{p}=0.015$, day 3: $g=0.126[0.003,0.304], \mathrm{p}=0.038)$. No significant differences between baseline and any post-test were found during days 4 and 5 . No significant difference between baseline and any post-test was found within the sham group for any day. By chance, there was a substantial difference in baseline uncrowded Landolt$\mathrm{C}$ visual acuity between the active and sham group (compare the dashed lines in Fig. 5-upper panel).

For the fellow eyes (Fig. 5-lower panel), there was a significant interaction between Group and Time, $\mathrm{F}_{3,45}=3.504 ; \mathrm{p}=0.023, \mathrm{\eta}_{\mathrm{p}}{ }^{2}=0.200$. No other omnibus main effects or interactions were significant. During day 1, the active group exhibited a significant improvement in contrast sensitivity from baseline for the post $5(g=0.817$ $[0.164,1.75], \mathrm{p}=0.035)$ and post $30(g=0.774[0.199,1.54], \mathrm{p}=0.02) \mathrm{min}$ measurements. No significant differences between baseline and any post-test were found for days 2-5. No significant differences between baseline and any post-test were found within the sham group for any day.

Crowded visual acuity. For the amblyopic eyes (Fig. 6-upper panel), there were no significant main effects or interactions (all $\mathrm{p}>0.05$ ). No significant changes from baseline were observed for any day for any group. As for uncrowded visual acuity, there was a substantial difference in baseline performance between the two groups that occurred by chance during randomization.

For the fellow eyes (Fig. 6-lower panel), there was a significant interaction between Group and Time, $\mathrm{F}_{3,45}=5.733 ; p=0.002, \eta_{p}{ }^{2}=0.291$. No other omnibus main effects or interactions were significant. During day 1 , the active group exhibited a significant improvement in crowded acuity from baseline for the post $5(g=0.404$ 


\begin{tabular}{|c|c|c|c|}
\hline & Mild & \multicolumn{2}{|l|}{ Moderate } \\
\hline \multicolumn{4}{|l|}{ Headache } \\
\hline Active $(\mathrm{N}=9)$ & 1 & \multicolumn{2}{|l|}{4} \\
\hline Sham $(N=8)$ & 2 & \multicolumn{2}{|l|}{0} \\
\hline \multicolumn{4}{|l|}{ Neck pain } \\
\hline Active $(\mathrm{N}=9)$ & 2 & \multicolumn{2}{|l|}{0} \\
\hline Sham $(N=8)$ & 1 & \multicolumn{2}{|l|}{0} \\
\hline \multicolumn{4}{|l|}{ Scalp pain } \\
\hline Active $(\mathrm{N}=9)$ & 1 & \multicolumn{2}{|l|}{0} \\
\hline Sham $(\mathrm{N}=8)$ & 0 & \multicolumn{2}{|l|}{0} \\
\hline \multicolumn{4}{|l|}{ Tingling sensation } \\
\hline Active $(\mathrm{N}=9)$ & 4 & \multicolumn{2}{|l|}{3} \\
\hline Sham $(N=8)$ & 6 & \multicolumn{2}{|l|}{1} \\
\hline \multicolumn{4}{|l|}{ Itching } \\
\hline Active $(\mathrm{N}=9)$ & 4 & \multicolumn{2}{|l|}{2} \\
\hline Sham $(N=8)$ & 5 & \multicolumn{2}{|l|}{0} \\
\hline \multicolumn{4}{|l|}{ Burning sensation } \\
\hline Active $(\mathrm{N}=9)$ & 2 & \multicolumn{2}{|l|}{3} \\
\hline Sham $(\mathrm{N}=8)$ & 1 & \multicolumn{2}{|l|}{0} \\
\hline \multicolumn{4}{|l|}{ Sleepiness } \\
\hline Active $(\mathrm{N}=9)$ & 2 & \multicolumn{2}{|l|}{1} \\
\hline Sham $(N=8)$ & 3 & \multicolumn{2}{|l|}{2} \\
\hline \multicolumn{4}{|l|}{ Trouble concentrating } \\
\hline Active $(\mathrm{N}=9)$ & 0 & \multicolumn{2}{|l|}{0} \\
\hline Sham $(N=8)$ & 1 & \multicolumn{2}{|l|}{0} \\
\hline \multicolumn{4}{|l|}{ Acute mood change } \\
\hline Active $(\mathrm{N}=9)$ & 0 & \multicolumn{2}{|l|}{0} \\
\hline Sham $(N=8)$ & 1 & \multicolumn{2}{|l|}{0} \\
\hline Others & Jaw stiffness & \multicolumn{2}{|c|}{ Neck tension } \\
\hline Active $(\mathrm{N}=9)$ & 1 & \multicolumn{2}{|l|}{1} \\
\hline Sham $(\mathrm{N}=8)$ & 0 & 0 & \\
\hline Any of the symptoms related to tES & Remote & Probable & Definite \\
\hline Active $(\mathrm{N}=9)$ & 0 & 3 & 6 \\
\hline Sham $(\mathrm{N}=8)$ & 2 & 0 & 6 \\
\hline
\end{tabular}

Table 2. Subjective experiences reported by participants after the day 5 active or sham tRNS session. No participants reported severe experiences. There were no statistically significant between group differences.

$[0.083,0.9], \mathrm{p}=0.05)$ and post $30 \mathrm{~min}(g=0.457[0.09,0.913], \mathrm{p}=0.039)$ measurements. During days 3 and 5 , the active group exhibited a significant improvement in crowded acuity from baseline to post $5 \mathrm{~min}(g=0.389$ $[0.065,1.06], p=0.007)$ and post $30 \mathrm{~min}(g=0.721[0.047,1.4], p=0.044)$ respectively. No significant differences between baseline and any post-test were found for days 2 and 4 . No significant differences between baseline and any post-test were found for the sham group.

Cumulative and long-term effects of tRNS. For the amblyopic eyes, there was a significant interaction between Group and Baseline for uncrowded visual acuity $\left(\mathrm{F}_{5,65}=3.372 ; p=0.009, \eta_{p}{ }^{2}=0.206\right)$. Pairwise comparisons for the active group revealed a significant difference between the day 1 baseline and the day $3(g=0.372$ $[0.163,0.771], \mathrm{p}=0.011)$, day $4(g=0.461[0.243,0.93], \mathrm{p}<0.003)$, day $5(g=0.369[0.065,0.809], \mathrm{p}=0.034)$, and day $28(g=0.454[0.219,1.03], p=0.003)$ baselines. No pairwise comparisons were significant for the sham group. There were no significant interactions for amblyopic eye contrast sensitivity or crowded visual acuity measurements or for any of the fellow eye measurements.

\section{Discussion}

Our results partially supported our experimental hypothesis that five daily sessions of visual cortex tRNS would improve amblyopic eye contrast sensitivity as well as crowded and uncrowded visual acuity in adult patients. We observed tRNS-induced improvements in contrast sensitivity and uncrowded visual acuity relative to the sham group for both amblyopic and fellow eyes. Crowded visual acuity improved for the fellow but not the amblyopic eyes. Across all outcome measures, pairwise comparisons revealed that acute tRNS effects were statistically significant on day 1 but became non-significant for later sessions. Only amblyopic eye uncrowded visual acuity exhibited a lasting effect of tRNS at follow-up. Our discussion will focus primarily on the results for contrast sensitivity 

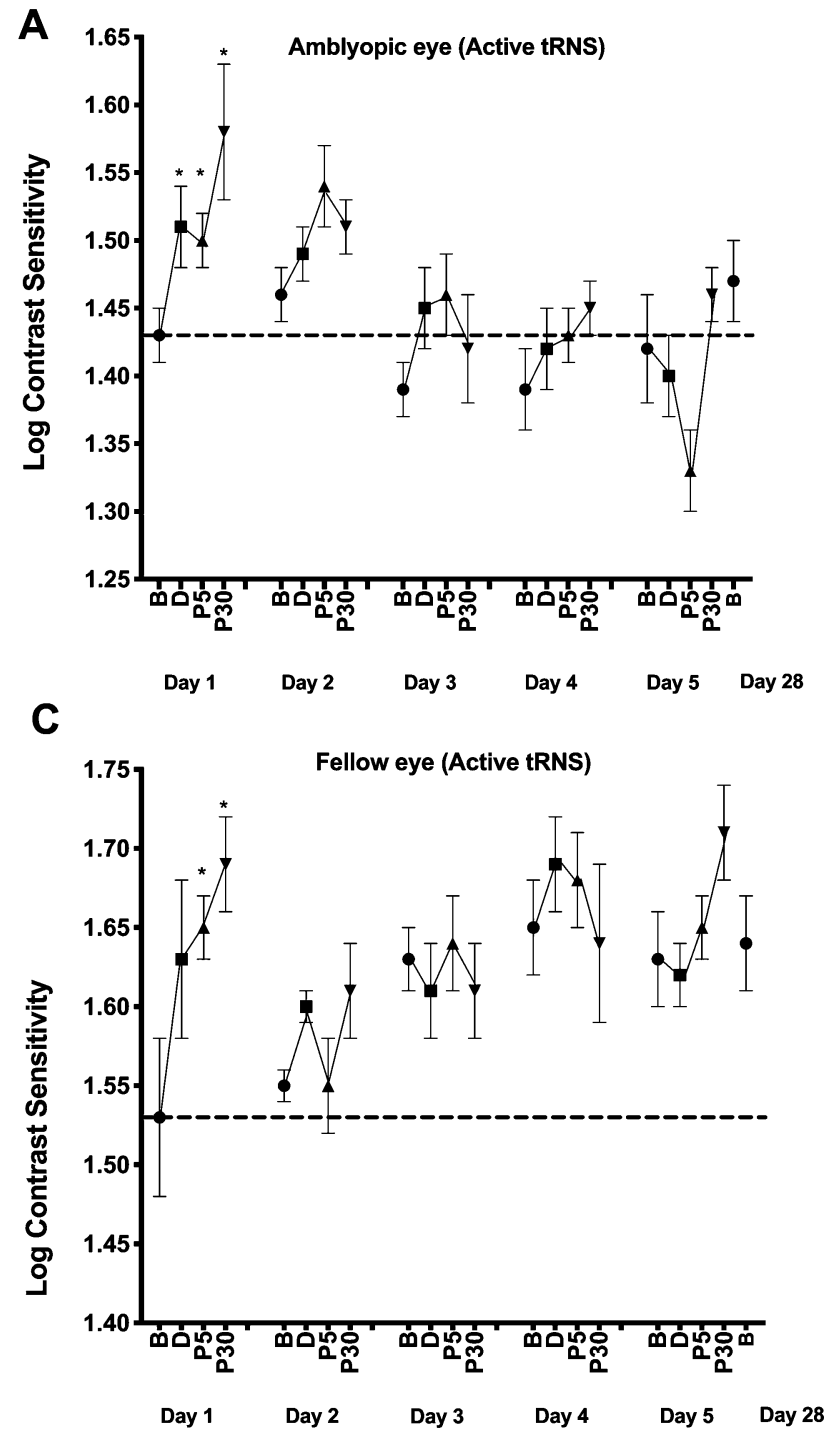

B

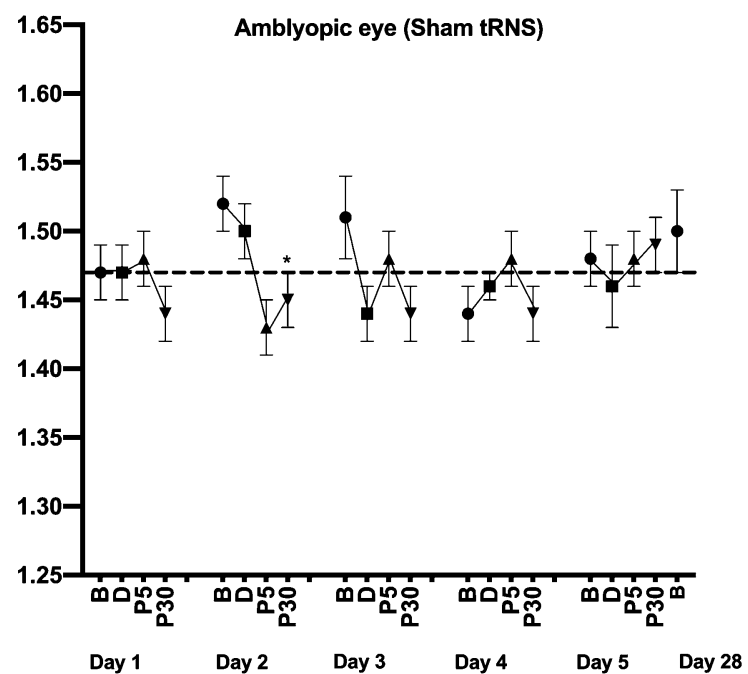

D

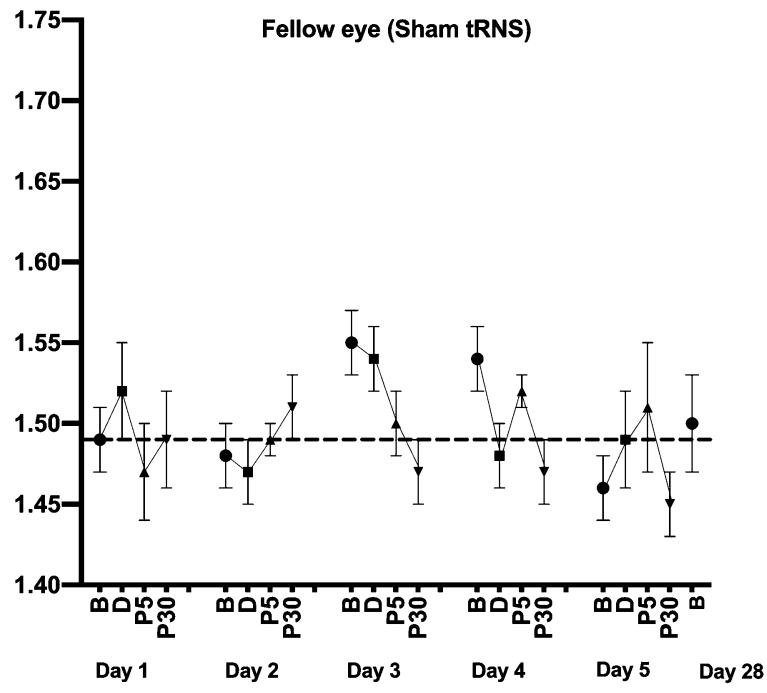

Figure 3. The effects of tRNS on contrast sensitivity during each daily session and at the day 28 follow-up visit. Data are shown separately for the amblyopic ( $\mathbf{A}$ and $\mathbf{B})$ and fellow $(\mathbf{C}$ and $\mathbf{D})$ eyes and for the active (A and C) and control (B and D) groups at baseline and during, $5 \mathrm{~min}$ (P5) and $30 \mathrm{~min}$ (P30) post tRNS. ${ }^{\star S}$ tatistically significant difference from baseline $(\mathrm{p}<0.05)$. Error bars show within-subject standard error of the mean $(\mathrm{SEM})$. The dashed horizontal lines represent the mean before-stimulation threshold on day 1. Larger y-axis values indicate better contrast sensitivity.

because initial baseline performance was matched between the groups. There were pronounced between-group baseline differences for amblyopic eye uncrowded and crowded visual acuity that occurred by chance during the randomization procedure (randomization occurred before baseline measures were conducted). The difference in baseline performance for the acuity outcome measures make it difficult to properly segregate tRNS effects from task learning effects. One reason for these baseline differences might be a difference in the proportion of patients with anisometropic amblyopia in active (78\%) and sham (50\%) groups. However, a much larger scale study will be required to determine whether amblyopia subtype influences the response to visual cortex tRNS.

tRNS-induced improvements in contrast sensitivity. Our observation that visual cortex tRNS improved amblyopic eye contrast sensitivity is consistent with a growing literature reporting improved contrast sensitivity, visual acuity, stereopsis, and an enhanced cortical response to amblyopic eye inputs following non-invasive visual cortex stimulation in adults with amblyopia ${ }^{26,39-42,70-72}$. A number of potential mechanisms have been proposed for tRNS effects. These include stochastic resonance and changes in the resting membrane potential $^{22,52}$. Stochastic resonance refers to an improvement in signal to noise ratio when a certain amount of noise (in this case neural noise induced by tRNS) is added to non-linear systems ${ }^{54}$. A number of psychophysical studies have provided compelling evidence that stochastic resonance occurs during visual cortex tRNS ${ }^{24,73-75}$. It is possible that the during-stimulation improvements we observed on day 1 for amblyopic eye contrast sensitiv- 


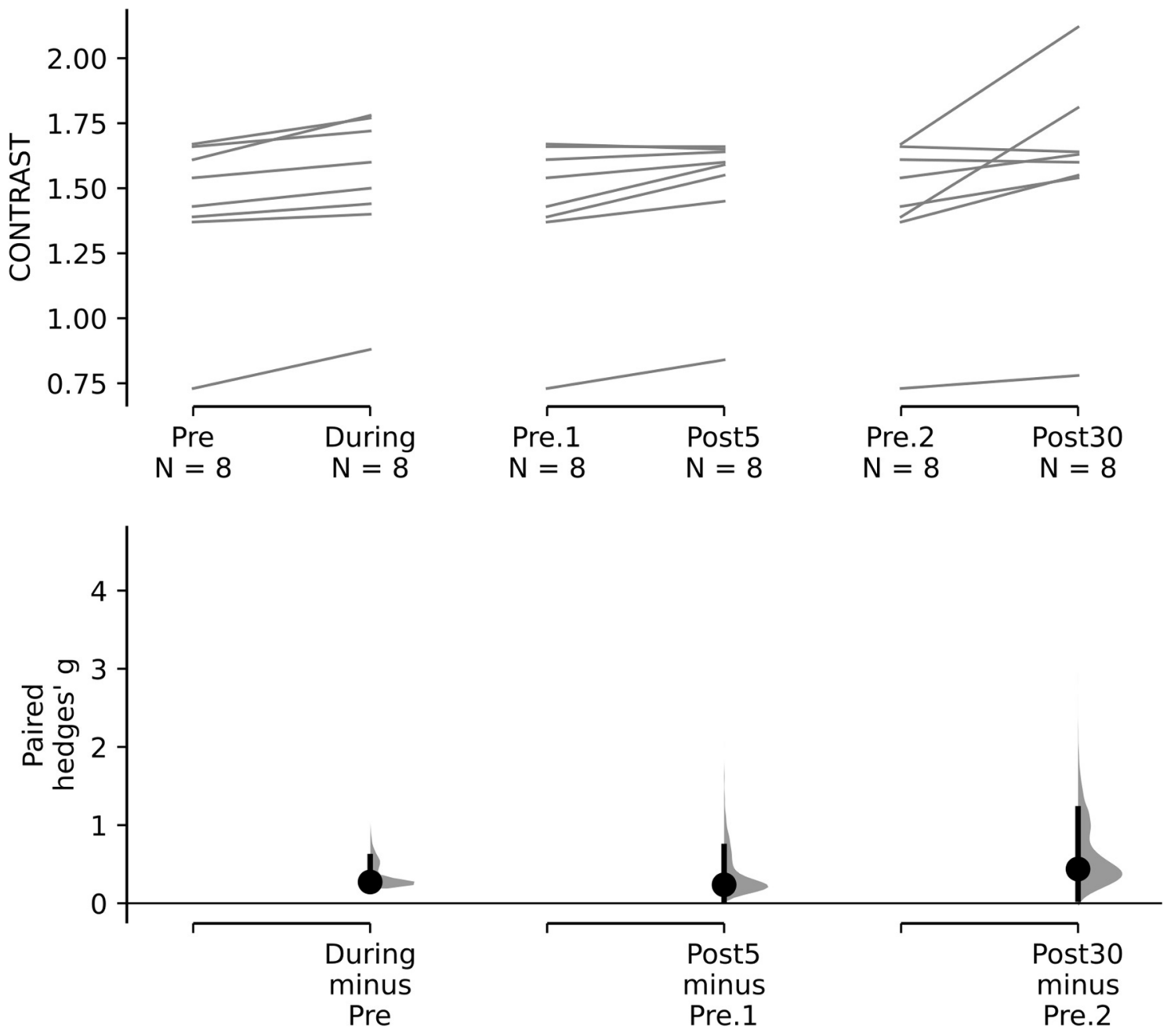

Figure 4. Paired Hedges' $g$ for three comparisons (during stimulation, Post $5 \mathrm{~min}$, Post $30 \mathrm{~min}$ ) to pre-test contrast sensitivity are shown for the active group amblyopic eye contrast sensitivity data using a Cumming estimation plot. Raw contrast threshold data for each participant are plotted on the upper axes; each paired set of observations is connected by a line. On the lower axes, paired Hedges's $g$ is plotted as a bootstrap sampling distribution. Hedge's $g$ value is depicted as dots; $95 \%$ confidence intervals are indicated by the ends of the vertical error bars.

ity were due to stochastic resonance. However, tRNS aftereffects (i.e. effects that outlast the duration of stimulation) cannot easily be explained by stochastic resonance.

Terney et al., proposed that increased motor cortex excitability following tRNS is related to the activity of sodium channels within the neural membrane ${ }^{76}$. Specifically, they proposed that tRNS may cause repetitive membrane depolarization that is sufficient to repeatedly open sodium channels but sub-threshold for generating an action potential. These synchronized local depolarizations where further hypothesized to induce lasting long-term potentiation-like effects at the level of individual neurons. However, a subsequent study found that pharmacological manipulation of NDMA receptors had no effect on tRNS aftereffects, whereas the GABA agonist lorazepam and carbamazepine, a sodium channel blocker, attenuated tRNS aftereffects ${ }^{77}$. These results are not consistent with a mechanism related to long-term potentiation but do support the involvement of sodium channels. Alternative mechanisms for the effects of electrical stimulation have also been proposed including regional increases in cortical blood flow $^{36}$, modified brain connectivity ${ }^{78}$ and changes in neurotransmitter concentration ${ }^{46,79}$. The precise underlying mechanism for the tRNS aftereffects we observed remains to be determined.

A previous study ${ }^{36}$ reporting improved amblyopic eye contrast sensitivity following both excitatory and inhibitory visual cortex rTMS proposed a mechanism linked to cortical homeostasis. According to this hypothesis, excitatory stimulation has a more pronounced effect on weakly activated/suppressed neural populations whereas inhibitory stimulation has a greater effect on strongly activated populations. Therefore, both excitatory and inhibitory stimulation are capable of restoring a level of homeostasis to the amblyopic visual cortex by reducing the difference in activation between amblyopic eye dominated neurons (weak activation/suppression) and fellow eye dominated neurons (strong activation) ${ }^{41}$. This, in turn, reduces suppression and/or the relative attenuation 

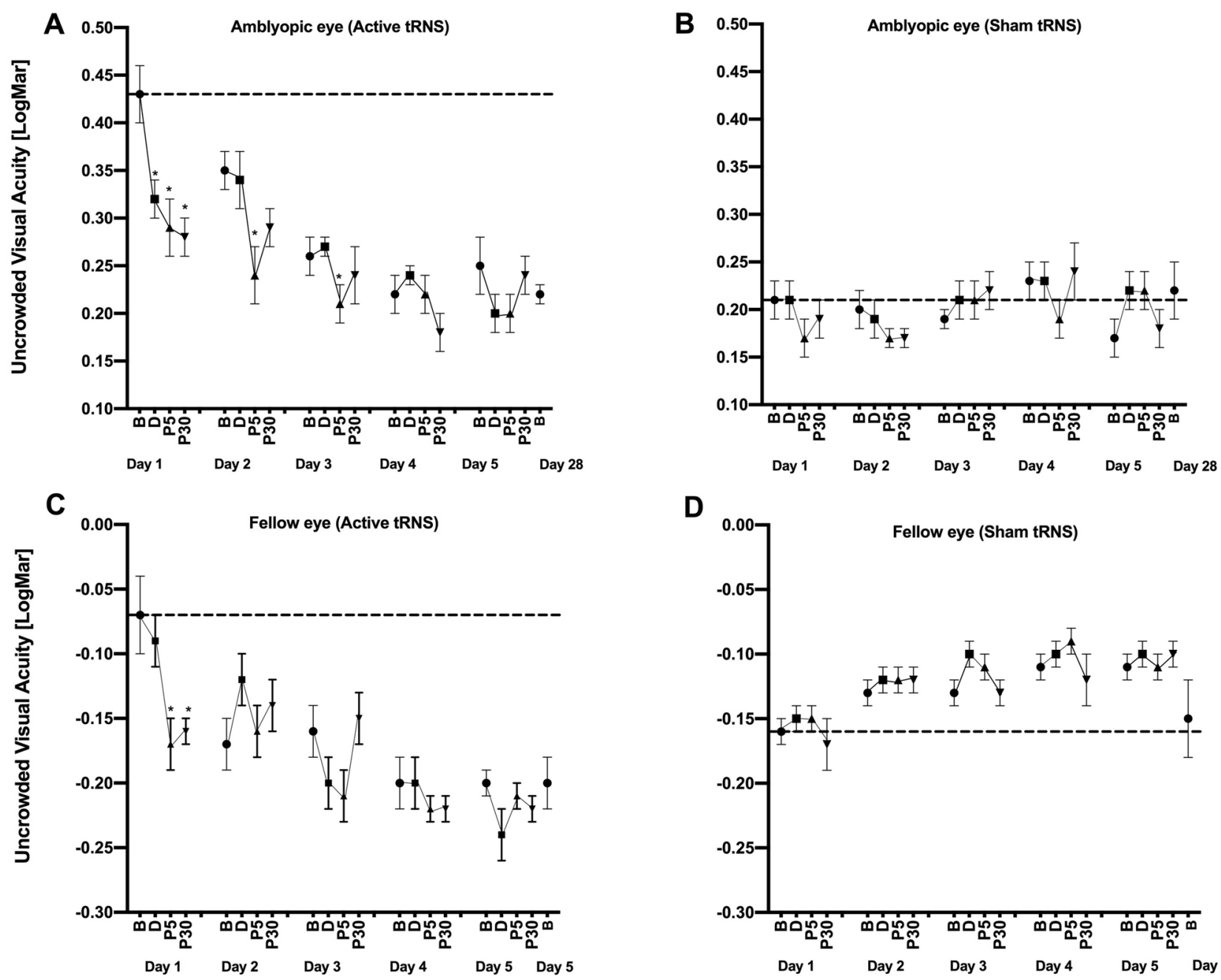

D

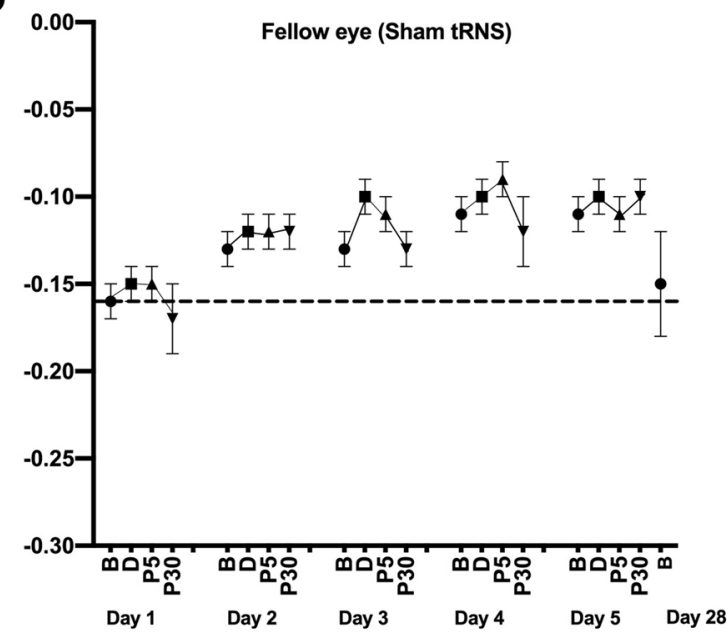

Figure 5. The effects of tRNS on uncrowded visual acuity during each daily session and at the day 28 follow-up visit. Data are shown as in Fig. 3. Lower (smaller/more negative) y-axis values indicate better uncrowded visual acuity.

of amblyopic-eye-driven neural activity. It is plausible that the excitatory tRNS we employed in this study acts through a homeostatic mechanism.

We also observed improved fellow eye contrast sensitivity in the tRNS group relative to the sham group. Non-invasive visual cortex stimulation studies have reported varying fellow eye effects. Studies using inhibitory stimulation protocols ( $1 \mathrm{~Hz}$ rTMS and continuous theta burst stimulation; cTBS) have reported reduced fellow eye contrast sensitivity ${ }^{35,36}$ whereas those using excitatory protocols (anodal tDCS and tRNS) ${ }^{24,42}$, including the present study, observed improvements. This pattern of results is consistent with the homeostasis hypothesis which predicts relatively impaired fellow eye function following inhibitory stimulation and does not rule out improved fellow eye function following excitatory stimulation. This is because excitatory effects may still occur within neuronal populations dominated by the fellow eye, just to a lesser extent than those dominated by the amblyopic eye.

Successive and cumulative tRNS effects on contrast sensitivity. A day by day analysis of the contrast sensitivity data revealed that tRNS effects were pronounced for both eyes on day 1 . However, the withinsession tRNS effects waned across sessions, becoming non-significant by day 2 for both eyes. This reduction in within-session tRNS effects was accompanied by stable session to session baseline performance indicating the absence of a cumulative tRNS effect on contrast sensitivity. The waning of within-session effects is consistent with Clavagnier et al.s ${ }^{40}$ study of repeated cTBS sessions in amblyopia, however cTBS did induce cumulative effects that improved baseline performance across sessions. One possible explanation for the waning of withinsession tRNS effects and the absence of a cumulative effect on contrast sensitivity relates to stimulation intensity. The relationship between tRNS intensity and visual function improvement during stimulation is an "inverted U", whereby stimulation that is weaker or stronger than an optimum level has limited effects ${ }^{52,53}$. Lasting changes in cortical excitability induced by prior sessions of tRNS might shift the optimal stimulation intensity towards lower levels, causing a waning of tRNS effects across sessions if stimulation intensity remains constant. If this is the case, tapering stimulation intensity across sessions would be a possible solution. 
A
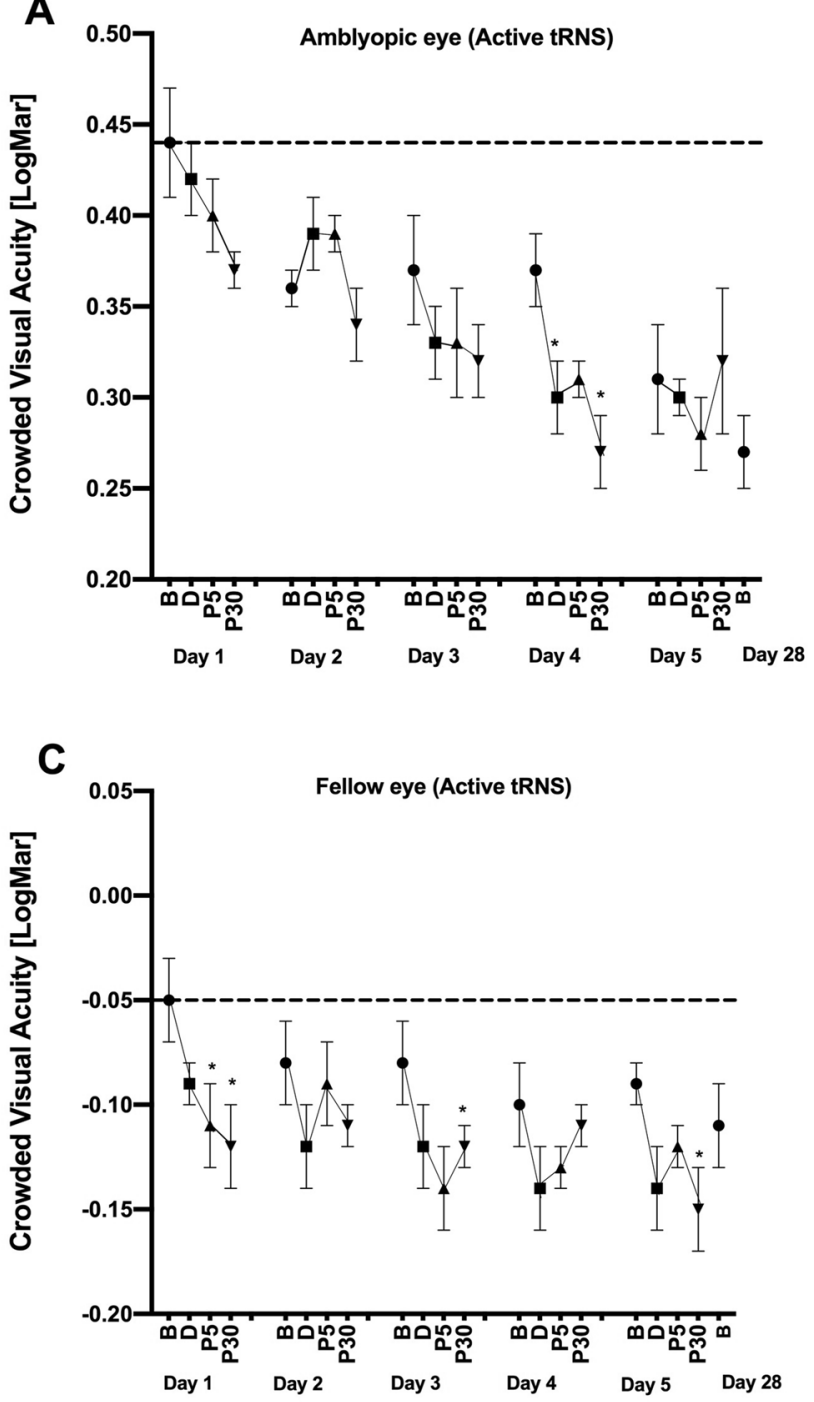

B

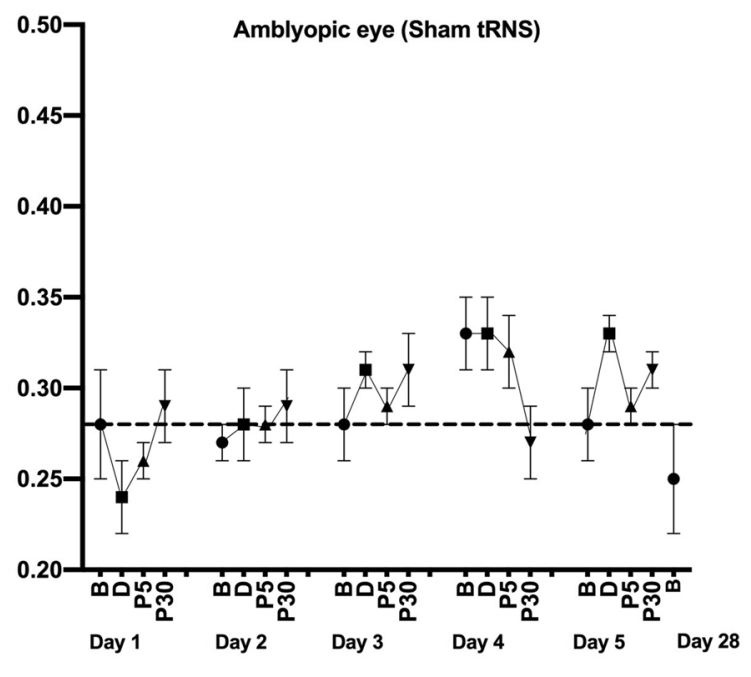

Figure 6. The effects of tRNS on crowded visual acuity during each daily session and at the day 28 follow-up visit. Data are shown as in Fig. 3. Lower (smaller/more negative) y-axis values indicate better crowded visual acuity.

Another possible explanation for an effect on day 1 and not subsequent days is a placebo effect. Although this cannot be completely ruled out, the use of a single masked, between subjects design combined with automated collection of outcome measures was intended to minimize this source of bias. In addition, participant reported sensations did not differ significantly between the two groups suggesting the adequate masking was preserved throughout the study.

tRNS effects of crowded and uncrowded visual acuity. It is not possible to draw strong conclusions relating to the amblyopic eye datasets for crowded and uncrowded visual acuity because there were large between-group differences in baseline performance that occurred by chance. However, baseline group differences were minimal for the fellow eye datasets and the results followed those for contrast sensitivity very closely; significant differences between groups that were characterized by within-session improvements early in the experiment and a gradual waning of tRNS effects. This suggests that transient tRNS effects occur for a range of visual functions and that long lasting effects may occur for uncrowded visual acuity.

Study limitations. The primary limitation of this study is the relatively small sample size. However, there is no indication in our data that the lack of long-term effects of visual cortex tRNS on amblyopic eye contrast sensitivity is due to insufficient statistical power. We had sufficient power to detect an effect of tRNS on day 1 and this effect waned across subsequent sessions. The small sample size did preclude the use of stratification for amblyopia subtype and baseline clinical characteristics within our randomization procedure and this likely contributed to the between group differences in baseline amblyopic eye visual acuity that are present in our data. 


\section{Conclusions}

tRNS can induce short-term contrast sensitivity improvements in adult amblyopic eyes, however repeated sessions of tRNS with a fixed set of stimulation parameters do not lead to enhanced or long-lasting effects. In agreement with previous non-invasive brain stimulation studies, these results demonstrate considerable short-term plasticity within the visual cortex of human adults with amblyopia and identify new pathways for future research such as the modification of stimulation parameters across sessions to maximize cumulative stimulation effects and the exploration of specific rather than random stimulation frequency bands.

Received: 11 August 2020; Accepted: 10 December 2020

Published online: 04 February 2021

\section{References}

1. Attebo, K. et al. Prevalence and cause of amblyopia in an adult population. Ophthalmology https://doi.org/10.1016/S0161 -6420(98)91862-0 (1998).

2. Webber, A. L. \& Wood, J. Amblyopia: Prevalence, natural history, functional effects and treatment. Clin. Exp. Optom. https://doi. org/10.1111/j.1444-0938.2005.tb05102.x (2005).

3. Pai, A. S. I. et al. Amblyopia prevalence and risk factors in Australian preschool children. Ophthalmology. https://doi.org/10.1016/j. ophtha.2011.06.024 (2012).

4. Asper, L., Crewther, D. \& Crewther, S. G. Strabismic amblyopia: Part 1: Psychophysics. Clin. Exp. Optom. https://doi. org/10.1111/j.1444-0938.2000.tb04892.x (2000).

5. Hamm, L. M., Black, J., Dai, S. \& Thompson, B. Global processing in amblyopia: A review. Front. Psychol. 5, 583. https://doi. org/10.3389/fpsyg.2014.00583 (2014).

6. McKee, S. P., Levi, D. M. \& Movshon, J. A. The pattern of visual deficits in amblyopia. J. Vis. 3, 5. https://doi.org/10.1167/3.5.5 (2003).

7. Levi, D. M. \& Klein, S. Hyperacuity and amblyopia. Nature https://doi.org/10.1038/298268a0 (1982).

8. Levi, D. M. \& Klein, S. A. Vernier acuity, crowding and amblyopia. Vis. Res. https://doi.org/10.1016/0042-6989(85)90208-1 (1985).

9. Stager, D. R., Everett, M. E. \& Birch, E. E. Comparison of crowding bar and linear optotype acuity in amblyopia. Am. Orthopt. J. https://doi.org/10.1080/0065955X.1990.11981816 (1990).

10. Bradley, A. \& Freeman, R. D. Contrast sensitivity in anisometropic amblyopia. Investig. Ophthalmol Vis. Sci. 21, 467 (1981).

11. Kiorpes, L., Kiper, D. C. \& Movshon, J. A. Contrast sensitivity and vernier acuity in amblyopic monkeys. Vis. Res. https://doi. org/10.1016/0042-6989(93)90107-8 (1993).

12. Hess, R. F. \& Howell, E. R. The threshold contrast sensitivity function in strabismic amblyopia: Evidence for a two type classification. Vis. Res. https://doi.org/10.1016/0042-6989(77)90009-8 (1977).

13. Birch, E. E. Amblyopia and binocular vision. Prog. Retin. Eye Res. https://doi.org/10.1016/j.preteyeres.2012.11.001 (2013).

14. Levi, D. M., Knill, D. C. \& Bavelier, D. Stereopsis and amblyopia: A mini-review. Vis. Res. https://doi.org/10.1016/j.visre s.2015.01.002 (2015).

15. Black, J. M., Hess, R. F., Cooperstock, J. R., To, L. \& Thompson, B. The measurement and treatment of suppression in amblyopia. J. Vis. Exp. https://doi.org/10.3791/3927 (2012).

16. Li, J. et al. The role of suppression in amblyopia. Investig. Ophthalmol. Vis. Sci. 52, 4169-4176. https://doi.org/10.1167/iovs.11-7233 (2011).

17. Hess, R. F. \& Thompson, B. Amblyopia and the binocular approach to its therapy. Vis. Res. 114, 4-16. https://doi.org/10.1016/j. visres.2015.02.009 (2015).

18. Kiorpes, L. \& Daw, N. Cortical correlates of amblyopia. Vis. Neurosci. 35, E016. https://doi.org/10.1017/S0952523817000232 (2018).

19. Loudon, S. E., Polling, J. R. \& Simonsz, H. J. Electronically measured compliance with occlusion therapy for amblyopia is related to visual acuity increase. Graefe's Arch. Clin. Exp. Ophthalmol. https://doi.org/10.1007/s00417-002-0570-z (2003).

20. Holmes, J. M. et al. Effect of age on response to amblyopia treatment in children. Arch. Ophthalmol. https://doi.org/10.1001/archo phthalmol.2011.179 (2011)

21. Inukai, Y. et al. Comparison of three non-invasive transcranial electrical stimulation methods for increasing cortical excitability. Front. Hum. Neurosci. 10, 668. https://doi.org/10.3389/fnhum.2016.00668 (2016).

22. Terney, D., Chaieb, L., Moliadze, V., Antal, A. \& Paulus, W. Increasing human brain excitability by transcranial high-frequency random noise stimulation. J. Neurosci. 28, 14147 (2008).

23. Paulus, W. Transcranial electrical stimulation (tES - tDCS; tRNS, tACS) methods. Neuropsychol. Rehabil. 21, 602-617. https://doi. org/10.1080/09602011.2011.557292 (2011).

24. Moret, B. et al. Differential effects of high-frequency transcranial random noise stimulation (hf-tRNS) on contrast sensitivity and visual acuity when combined with a short perceptual training in adults with amblyopia. Neuropsychologia 114, 125-133. https:// doi.org/10.1016/j.neuropsychologia.2018.04.017 (2018).

25. Camilleri, R., Pavan, A. \& Campana, G. The application of online transcranial random noise stimulation and perceptual learning in the improvement of visual functions in mild myopia. Neuropsychologia 89, 225-231. https://doi.org/10.1016/j.neuropsychologi a.2016.06.024 (2016).

26. Spiegel, D. P. et al. Transcranial direct current stimulation enhances recovery of stereopsis in adults with amblyopia. Neurotherapeutics 10, 831-839. https://doi.org/10.1007/s13311-013-0200-y (2013).

27. O'Brien, A. T. et al. Non-invasive brain stimulation for fine motor improvement after stroke: A meta-analysis. Eur. J. Neurol. https ://doi.org/10.1111/ene.13643 (2018).

28. Norise, C. \& Hamilton, R. H. Non-invasive brain stimulation in the treatment of post-stroke and neurodegenerative aphasia: Parallels, differences, and lessons learned. Front. Hum. Neurosci. 10, 1-16. https://doi.org/10.3389/fnhum.2016.00675 (2017).

29. Kubis, N. Non-invasive brain stimulation to enhance post-stroke recovery. Front. Neural Circuits 10, 56. https://doi.org/10.3389/ fncir.2016.00056 (2016).

30. Schlaug, G., Renga, V. \& Nair, D. Transcranial direct current stimulation in stroke recovery. Arch. Neurol. 65, 1571-1576. https:// doi.org/10.1001/archneur.65.12.1571 (2008).

31. Hu, X.-S. et al. Feasibility of non-invasive brain modulation for management of pain related to chemoradiotherapy in patients with advanced head and neck cancer. Front. Hum. Neurosci. 10, 466. https://doi.org/10.3389/fnhum.2016.00466 (2016).

32. Palm, U. et al. Effects of transcranial random noise stimulation (tRNS) on affect, pain and attention in multiple sclerosis. Restor. Neurol. Neurosci. 34, 189-199. https://doi.org/10.3233/RNN-150557 (2016).

33. Vanneste, S., Fregni, F. \& De Ridder, D. Head-to-head comparison of transcranial random noise stimulation, transcranial AC stimulation, and transcranial DC stimulation for tinnitus. Front. Psychiatry https://doi.org/10.3389/fpsyt.2013.00158 (2013).

34. Pal, N., Maire, R., Stephan, M. A., Herrmann, F. R. \& Benninger, D. H. Transcranial direct current stimulation for the treatment of chronic tinnitus: A randomized controlled study. Brain Stimul. 8, 1101-1107. https://doi.org/10.1016/j.brs.2015.06.014 (2015). 
35. Perin, C. et al. Non-invasive current stimulation in vision recovery: A review of the literature. Restor. Neurol. Neurosci. https://doi. org/10.3233/rnn-190948 (2019).

36. Sabel, B. A. et al. Vision modulation, plasticity and restoration using non-invasive brain stimulation-An IFCN-sponsored review. Clin. Neurophysiol. https://doi.org/10.1016/j.clinph.2020.01.008 (2020).

37. Sabel, B. A. et al. Non-invasive alternating current stimulation improves vision in optic neuropathy. Restor. Neurol. Neurosci. 29, 493-505. https://doi.org/10.3233/RNN-2011-0624 (2011).

38. Gall, C. et al. Alternating current stimulation for vision restoration after optic nerve damage: A randomized clinical trial. PLoS ONE. https://doi.org/10.1371/journal.pone.0156134 (2016).

39. Thompson, B., Mansouri, B., Koski, L. \& Hess, R. F. Brain plasticity in the adult: Modulation of function in amblyopia with rTMS. Curr. Biol. 18, 1067-1071. https://doi.org/10.1016/j.cub.2008.06.052 (2008).

40. Clavagnier, S., Thompson, B. \& Hess, R. F. Long lasting effects of daily theta burst rTMS sessions in the human amblyopic cortex. Brain Stimul. 6, 860-867. https://doi.org/10.1016/j.brs.2013.04.002 (2013).

41. Spiegel, D. P., Byblow, W. D., Hess, R. F. \& Thompson, B. Anodal transcranial direct current stimulation transiently improves contrast sensitivity and normalizes visual cortex activation in individuals with amblyopia. Neurorehabil. Neural Repair 27, 760-769. https://doi.org/10.1177/1545968313491006 (2013).

42. Ding, Z. et al. The effect of transcranial direct current stimulation on contrast sensitivity and visual evoked potential amplitude in adults with amblyopia. Sci. Rep. 6, 19280. https://doi.org/10.1038/srep19280 (2016).

43. Castaño-Castaño, S. et al. Transcranial direct current stimulation improves visual acuity in amblyopic Long-Evans rats. Brain Res. 1657, 340-346. https://doi.org/10.1016/j.brainres.2017.01.003 (2017).

44. Castaño-Castaño, S. et al. Transcranial direct-current stimulation (tDCS) improves detection of simple bright stimuli by amblyopic Long Evans rats in the SLAG task and produces an increase of parvoalbumin labelled cells in visual cortices. Brain Res. https:/doi. org/10.1016/j.brainres.2018.09.039 (2019).

45. Castaño-Castaño, S. et al. tDCS recovers depth perception in adult amblyopic rats and reorganizes visual cortex activity. Behav. Brain Res. https://doi.org/10.1016/j.bbr.2019.111941 (2019).

46. Bachtiar, V., Near, J., Johansen-Berg, H. \& Stagg, C. J. Modulation of GABA and resting state functional connectivity by transcranial direct current stimulation. Elife 4, 1-9. https://doi.org/10.7554/eLife.08789 (2015).

47. Sengpiel, F., Blakemore, C., Kind, P. C. \& Harrad, R. Interocular suppression in the visual cortex of strabismic cats. J. Neurosci. 14, 6855-6871. https://doi.org/10.1523/JNEUROSCI.14-11-06855.1994 (1994).

48. Hensch, T. K., Li, R. W., Bavelier, D., Levi, D. M. \& Dan, Y. Removing brakes on adult brain plasticity: From molecular to behavioral interventions. J. Neurosci. https://doi.org/10.1523/jneurosci.4812-10.2010 (2010).

49. Ho, K.-A., Taylor, J. \& Loo, C. P. Transcranial random noise stimulation: A new approach to stimulating the brain. Clin. Neurophysiol. https://doi.org/10.1016/j.clinph.2013.04.286 (2013).

50. Fertonani, A., Pirulli, C. \& Miniussi, C. Random noise stimulation improves neuroplasticity in perceptual learning. J. Neurosci. https://doi.org/10.1523/jneurosci.2002-11.2011 (2011).

51. Herpich, F. et al. Boosting learning efficacy with noninvasive brain stimulation in intact and brain-damaged humans. J. Neurosci. https://doi.org/10.1523/JNEUROSCI.3248-18.2019 (2019).

52. van der Groen, O. \& Wenderoth, N. Transcranial random noise stimulation of visual cortex: Stochastic resonance enhances central mechanisms of perception. J. Neurosci. 36, 5289 (2016).

53. Pavan, A. et al. Modulatory mechanisms underlying high-frequency transcranial random noise stimulation (hf-tRNS): A combined stochastic resonance and equivalent noise approach. Brain Stimul. https://doi.org/10.1016/J.BRS.2019.02.018 (2019).

54. McDonnell, M. D. \& Ward, L. M. The benefits of noise in neural systems: Bridging theory and experiment. Nat. Rev. Neurosci. https://doi.org/10.1038/nrn3061 (2011).

55. Antal, A. \& Herrmann, C. S. Transcranial alternating current and random noise stimulation: Possible mechanisms. Neural Plast. 2016, 1-12. https://doi.org/10.1155/2016/3616807 (2016).

56. Gao, T. Y. et al. Effectiveness of a binocular video game vs placebo video game for improving visual functions in older children, teenagers, and adults with amblyopia: A randomized clinical trial. JAMA Ophthalmol. 136, 172-181. https://doi.org/10.1001/jamao phthalmol.2017.6090 (2018).

57. Poreisz, C., Boros, K., Antal, A. \& Paulus, W. Safety aspects of transcranial direct current stimulation concerning healthy subjects and patients. Brain Res. Bull. 72, 208-214. https://doi.org/10.1016/j.brainresbull.2007.01.004 (2007).

58. Bikson, M. et al. Safety of transcranial direct current stimulation: Evidence based update 2016. Brain Stimul. 9, 641-661. https:// doi.org/10.1016/j.brs.2016.06.004 (2016).

59. Nitsche, M. A. et al. Transcranial direct current stimulation: State of the art 2008. Brain Stimul. https://doi.org/10.1016/j. brs.2008.06.004 (2008).

60. Bikson, M., Datta, A. \& Elwassif, M. Establishing safety limits for transcranial direct current stimulation. Clin. Neurophysiol. 120, 1033-1034. https://doi.org/10.1016/j.clinph.2009.03.018 (2009).

61. Bindman, L. J., Lippold, O. C. J. \& Redfearn, J. W. T. The action of brief polarizing currents on the cerebral cortex of the rat (1) during current flow and (2) in the production of long-lasting after-effects. J. Physiol. https://doi.org/10.1113/jphysiol.1964.sp007 425 (1964).

62. Nitsche, M. A. \& Paulus, W. Excitability changes induced in the human motor cortex by weak transcranial direct current stimulation. J. Physiol. 3, 633-639 (2000).

63. Antal, A., Nitsche, M. A. \& Paulus, W. External modulation of visual perception in humans. NeuroReport 12, 3553-3555. https:// doi.org/10.1097/00001756-200111160-00036 (2001).

64. Paneri, B. et al. Tolerability of repeated application of transcranial electrical stimulation with limited outputs to healthy subjects. Brain Stimul. 9, 740-754. https://doi.org/10.1016/j.brs.2016.05.008 (2016).

65. Nitsche, M. A. et al. Safety criteria for transcranial direct current stimulation (tDCS) in humans. Clin. Neurophysiol. 114, 22202222. https://doi.org/10.1016/S1388-2457(03)00235-9 (2003).

66. Bach, M. The "Freiburg visual acuity test"-Automatic measurement of visual acuity. Optom. Vis. Sci. 73, 49 (1996).

67. Bach, M. The Freiburg visual acuity test-variability unchanged by post-hoc re-analysis. Graefe's Arch. Clin. Exp. Ophthalmol. https ://doi.org/10.1007/s00417-006-0474-4 (2007).

68. R Development Core Team. R Development Core Team, R: A Language and Environment for Statistical Computing (2020).

69. Ho, J., Tumkaya, T., Aryal, S., Choi, H. \& Claridge-Chang, A. Moving beyond P values: Data analysis with estimation graphics. Nat. Methods. https://doi.org/10.1038/s41592-019-0470-3 (2019).

70. Thompson, B., Mansouri, B., Koski, L. \& Hess, R. F. From motor cortex to visual cortex: The application of noninvasive brain stimulation to amblyopia. Dev. Psychobiol. 54, 263-273. https://doi.org/10.1002/dev.20509 (2012).

71. Bocci, T. et al. Unilateral application of cathodal tDCS reduces transcallosal inhibition and improves visual acuity in amblyopic patients. Front. Behav. Neurosci. 12, 1-9. https://doi.org/10.3389/fnbeh.2018.00109 (2018).

72. Tuna, A. R. et al. Transcranial Magnetic Stimulation in Adults With Amblyopia. J. Neuroophthalmol. 40(2), 185-192. https://doi. org/10.1097/WNO.0000000000000828 (2020).

73. van Koningsbruggen, M. G., Ficarella, S. C., Battelli, L. \& Hickey, C. Transcranial random-noise stimulation of visual cortex potentiates value-driven attentional capture. Soc. Cogn. Affect. Neurosci. 11, 1481-1488. https://doi.org/10.1093/scan/nsw056 (2016). 
74. van der Groen, O., Mattingley, J. B. \& Wenderoth, N. Altering brain dynamics with transcranial random noise stimulation. Sci. Rep. https://doi.org/10.1038/s41598-019-40335-w (2019).

75. Herpich, F. et al. Transcranial random noise stimulation enhances visual learning in healthy adults. J. Vis. https://doi. org/10.1167/15.12.40 (2015).

76. Terney, D., Chaieb, L., Moliadze, V., Antal, A. \& Paulus, W. Increasing human brain excitability by transcranial high-frequency random noise stimulation. J. Neurosci. https://doi.org/10.1523/JNEUROSCI.4248-08.2008 (2008).

77. Chaieb, L., Antal, A. \& Paulus, W. Transcranial random noise stimulation-induced plasticity is NMDA-receptor independent but sodium-channel blocker and benzodiazepines sensitive. Front. Neurosci. https://doi.org/10.3389/fnins.2015.00125 (2015).

78. Bola, M. et al. Brain functional connectivity network breakdown and restoration in blindness. Neurology. https://doi.org/10.1212/ WNL.0000000000000672 (2014)

79. Stagg, C. J., Bachtiar, V. \& Johansen-Berg, H. The role of GABA in human motor learning. Curr. Biol. https://doi.org/10.1016/j. cub.2011.01.069 (2011).

\section{Acknowledgements}

This work was supported by CIHR Grant 390283, CFI Grant 34095 and NSERC Grants RPIN-05394 and RGPAS477166 to BT.

\section{Author contributions}

Conceptualization R.D. and B.T.; experimental design R.D., B.T., C.T. and M.W.D.; data collection R.D. and C.T.; data analysis R.D., A.S., A.P.J. and B.T.; data interpretation R.D., A.S., A.P.J. and B.T.; manuscript writing R.D. and B.T., revision and review of manuscript all authors.

\section{Competing interests}

The authors declare no competing interests.

\section{Additional information}

Correspondence and requests for materials should be addressed to B.T.

Reprints and permissions information is available at www.nature.com/reprints.

Publisher's note Springer Nature remains neutral with regard to jurisdictional claims in published maps and institutional affiliations.

(c) (1) Open Access This article is licensed under a Creative Commons Attribution 4.0 International License, which permits use, sharing, adaptation, distribution and reproduction in any medium or format, as long as you give appropriate credit to the original author(s) and the source, provide a link to the Creative Commons licence, and indicate if changes were made. The images or other third party material in this article are included in the article's Creative Commons licence, unless indicated otherwise in a credit line to the material. If material is not included in the article's Creative Commons licence and your intended use is not permitted by statutory regulation or exceeds the permitted use, you will need to obtain permission directly from the copyright holder. To view a copy of this licence, visit http://creativecommons.org/licenses/by/4.0/.

(C) The Author(s) 2021 